
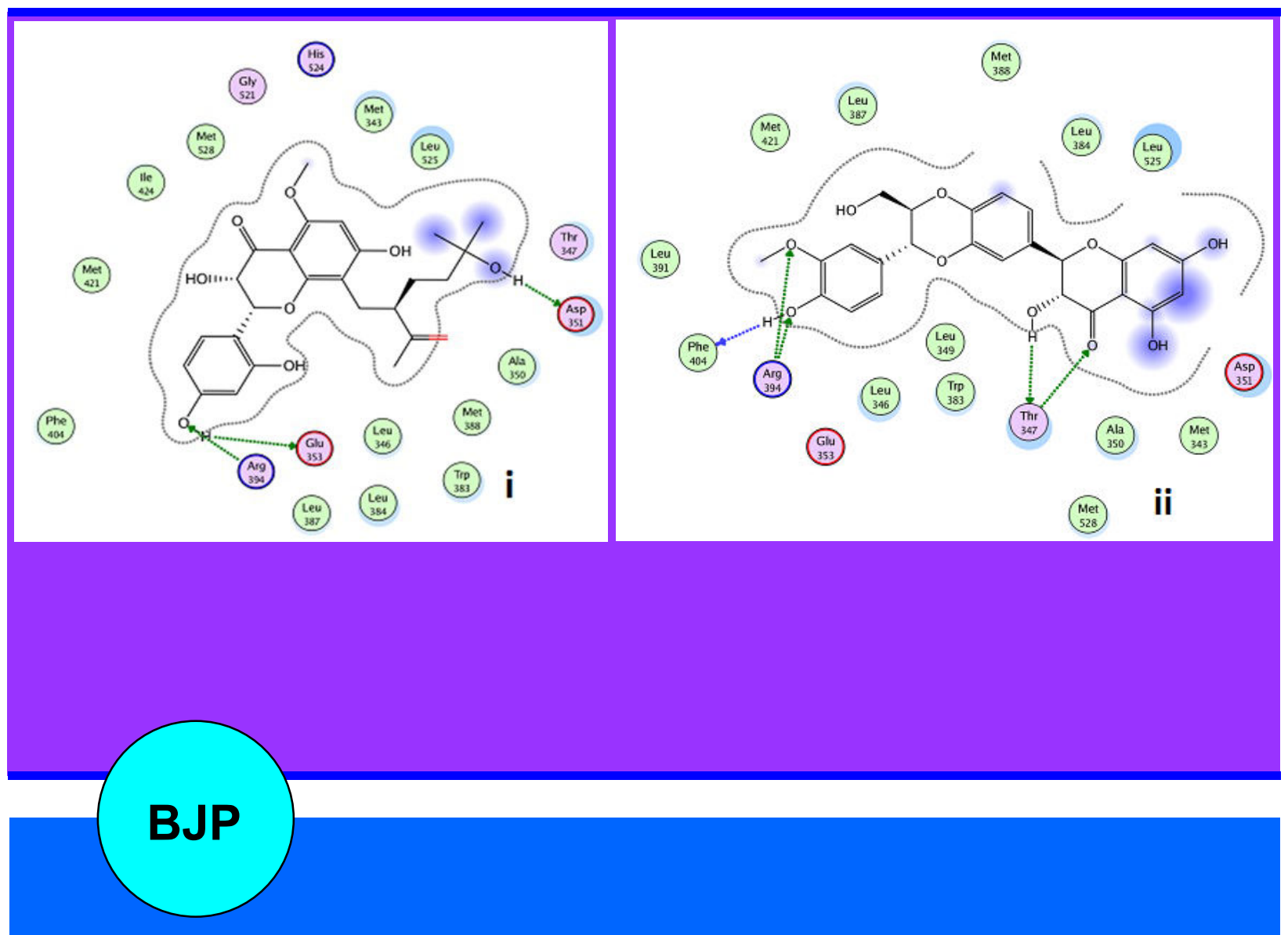

Bangladesh Journal of Pharmacology

Research Article

Anti-cancer potential of phytochemicals against breast cancer: Molecular docking and simulation approach 


\title{
Anti-cancer potential of phytochemicals against breast cancer: Molecular docking and simulation approach
}

\author{
Bilal Ahmed', Usman Ali Ashfaq1, Muhammad Tahir ul Qamar' and Matloob Ahmad' \\ ${ }^{1}$ Department of Bioinformatics and Biotechnology, Government College University (GCU), Faisalabad, Pakistan; \\ ${ }^{2}$ Department of Chemistry, Government College University, Faisalabad, Pakistan.
}

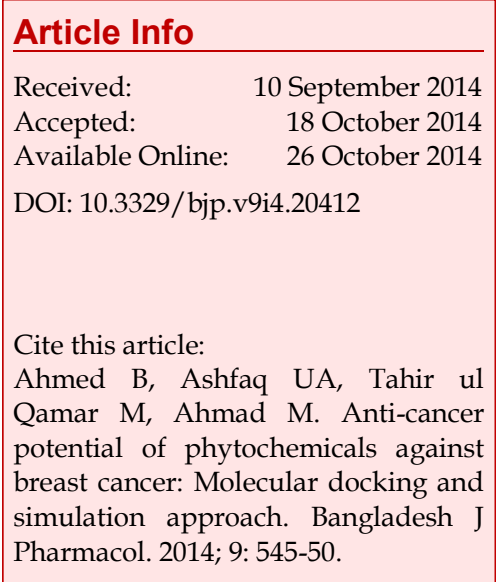

\section{Abstract}

Breast cancer malignancy is prevailing among the women not only from the developing countries but also from the developed one at the rate of $18 \%$ of total population worldwide. One of the main causes of breast cancer is estrogen receptor alpha. Overexpression of estrogen receptor is seen in number of cases of breast cancer. Tamoxifen was used as a reference drug in present study. Almost 80,000 species of plants are used as a source of medicines. Current study was totally based on the screening of phytochemicals to find out the biomolecules having strong bonding actions as compared to tamoxifen. Present study exhibited that 10 molecules (kushenol K, silybin, taxifolin 3-O-acetate, rosemarinic acid, secundifloran, kushenol N, kurarinol, podophyllotoxone, AC1LCW2L, leachianone G) have successful and potential binding with the target molecule as compared to tamoxifen. These molecules can be used for the treatment of breast cancer and birth control.

\section{Introduction}

In breast cancer, breast cells lose their normal control and start to proliferate at higher rate as compared to normal cells. Breast cancer is the most familiar form of cancer which affects the women's around the world and the second major form of cancer which is a cause of death next to the lung cancer. Rate of breast cancer is high in developed countries as compared to the developing countries (Sahu et al., 2011)

Number of molecular factors are determined which are used in diagnosis and remedy of breast cancer. Estrogen receptor alpha(ER-a) is most commonly used molecular marker for breast cancer. ER-a is the member of nuclear receptor family which controls number of physiological processes. Estrogen is the ligand of ER which activates the estrogen receptor. Overexpression of ER-a is seen in breast cancer (Holst et al., 2007). Ratio of ER positive breast cancers is sixty percent (Giacinti el al., 2006).

Medicinal plants and their extracts are used as a source of medicine. $25 \%$ of total medicines are taken from the plants in well developed countries while in developing countries rate is much higher (Thomas et al., 1998). Phytochemicals are molecules present in plants and control the number of diseases. Aim of this study was to screen out the effective bioactive compounds which may be potential inhibitors of ER-a in future and may act as a drug which may be effective in preventing the breast cancer. Tamoxifen used as control drug in present study.

\section{Materials and Methods}

A library of 4209 phytochemicals were docked counter to the estrogen receptor alpha with the help of docking software known as Molecular Operating System (MOE, 2013).

\section{Preparation of target}

Structure of estrogen receptor alpha was taken from the Protein Data Bank (PDB) by using its PDB ID which is 
"3ERT". For the refinement of target structure, removal of water molecules and 3D protonation was done with the help of MOE. Process of energy minimization was performed by using its standard parameters.

\section{Preparation of phytochemicals database}

All selected phyto-chemicals of different plants were downloaded from Pubchem (https:// pubchem.ncbi.nlm.nih.gov/), Maps database (Ashfaq et al., 2013) and MP3D (http://bioinform.info/). Each and every ligand molecule was saved into the MOE database after energy minimization.

\section{Molecular docking}

Binding Pocket containing the desired residue (ARG 394) was selected with the help of site finder tool of MOE. Following parameters were used to calculate the score and interaction of ligand molecules with the ER-a.

- London dG was used as a rescoring function.

- Placement : Triangle matcher

- Forcefield was used for refinement and liquid simulations.

- Retain : 10

- Rescoring was also done by using London $\mathrm{dG}$.

Most suitable interactions of ligand molecules with target were selected on the base of score and Root-Mean -Square Deviation (RMSD) values.

\section{Results}

Target structure was taken from PDB in 3D format. Energy minimization and other steps were performed to refine the structure. 4209 phytochemicals were taken from different plants belong to different classes and were docked against the estrogen receptor alpha.

According to the given command MOE gave the ten best confirmations of each ligand molecule. Confirmations were ranked on the base of docking score calculated by the MOE. Candidates containing the highest $S$ score were selected for further analysis. Top 10 molecules with highest docking score are given in (Table I).

Pubchem Id, other drug like properties of the selected candidates and interacting residues of estrogen receptor alpha with the selected molecules are also given in the (Table I). Structural formulas of best selected molecules are shown in (Figure 1).

Tamoxifen was used as a control drug. Tamoxifen is a commercially available drug which binds with the estrogen receptor and blocks its function and prevents the proliferation of breast cells or in other words prevents from breast cancer. Tamoxifen showed binding interactions with an active residue of target molecule Arg394. Docking score of tamoxifen with the target molecule was -13.9701 . Other properties of reference drug are given in (Table I). Interactions are shown in (Figure 2) and binding mode of drug with target is given in (Figure 3).

Further interactions of selected molecules were studied. Silybin is a biomolecule found in seeds of "Milk histle"(Silybum marianum) with molecular formula $\mathrm{C}_{25} \mathrm{H}_{22} \mathrm{O}_{10}$ had shown strong bonding with Arg394, Thr347 and Glu353 residues of receptor molecule as well as showed less docking score(-16.7010) as compared to the reference drug (-13.9701). All other selected phytochemicals like (kushenol K, taxifolin 3-O-acetate, etc) are summarized and residues which interacts with these molecules are also given in (Table I). Interactions of active residues of receptor with top five molecules

Table I

Pubchem ID, docking score and other drug like properties of finalized molecules

\begin{tabular}{|lrrrrrrl|}
\hline SL. & Pubchem_ID & Docking score & $\begin{array}{c}\text { Molecular } \\
\text { weight }\end{array}$ & $\begin{array}{c}\text { Donors } \\
\text { No. }\end{array}$ & $\begin{array}{c}\text { Accep- } \\
\text { tors }\end{array}$ & $\begin{array}{c}\text { Log } \\
\text { value }\end{array}$ & Residues \\
\hline I & 44428630 & -17.9303 & 472.534 & 5 & 8 & 3.871 & Arg394, Asp351, Glu353 \\
II & 31553 & -16.7010 & 482.441 & 5 & 10 & 2.554 & Arg394, Thr347, Phe404 \\
III & 442540 & -16.6179 & 346.291 & 4 & 7 & 1.853 & Arg394, Thr347, Glu353 \\
IV & 5315615 & -16.5006 & 360.318 & 5 & 7 & 1.761 & Arg394, Glu353, Thr347, Phe404 \\
V & 10091530 & -15.4800 & 386.400 & 4 & 7 & 3.050 & Arg394, Thr347 \\
VI & 381851 & -15.4598 & 454.519 & 4 & 7 & 4.676 & Arg394, Leu387 \\
VII & 44563198 & -15.2642 & 456.535 & 4 & 7 & 4.900 & Arg394, Leu387, Asp351 \\
VIII & 443014 & -15.2458 & 412.394 & 0 & 7 & 2.559 & Arg394, Thr347 \\
IX & 638288 & -15.1743 & 316.309 & 3 & 6 & 2.264 & Arg394, Thr347 \\
X & 5275227 & -15.0781 & 356.374 & 4 & 6 & 3.820 & Arg394 \\
XI & Tamoxifen & -13.9701 & 387.513 & 1 & 3 & 5,519 & Arg394 \\
\hline
\end{tabular}



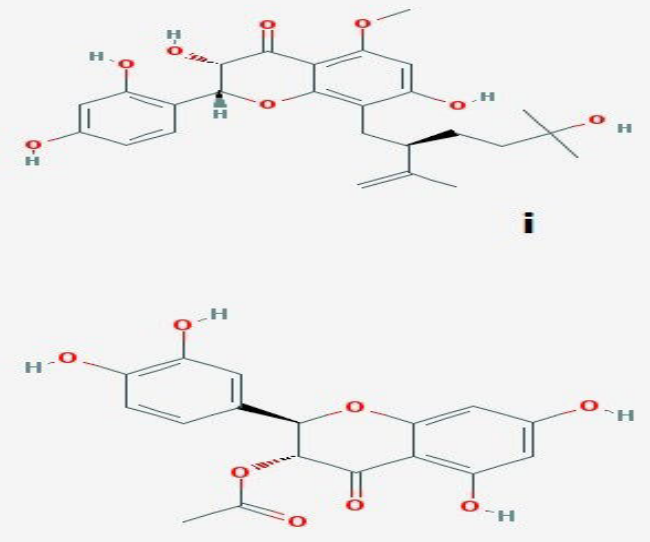

iii
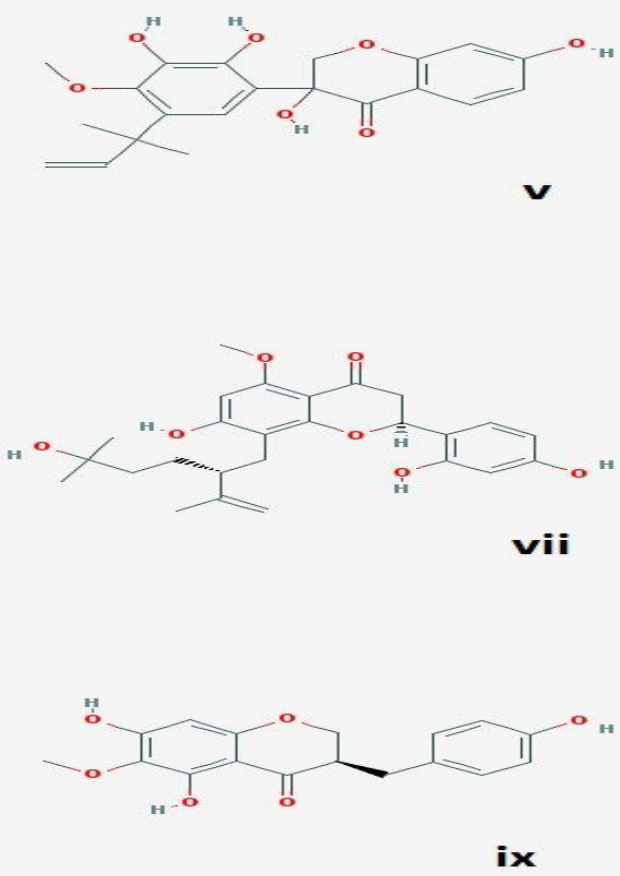

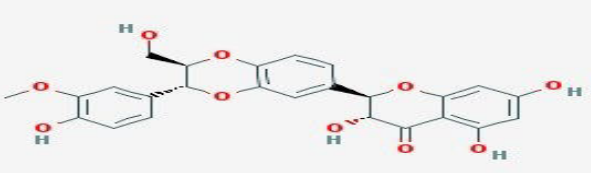

ii

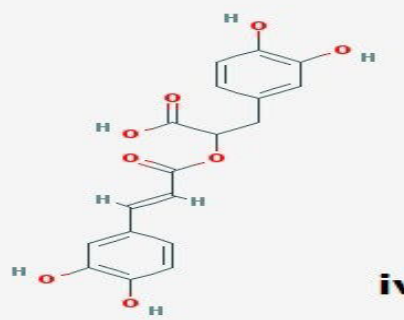

iv 운
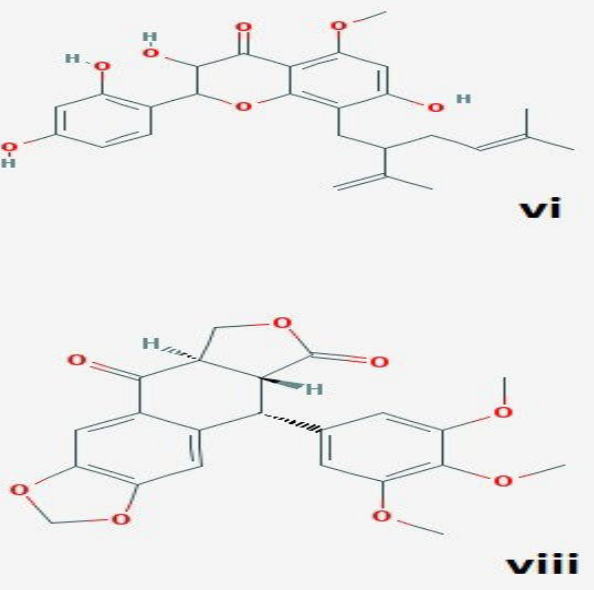

viii

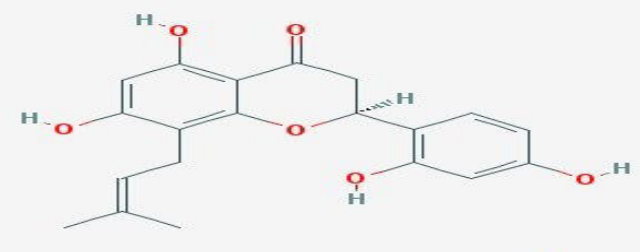

Figure 1: Structures of 10 best selected ligand molecules

and reference drug are given in (Figure 2) and best binding modes of top five molecules and tamoxifen are shown in (Figure 3).

All the finalized molecules were assessed for Lipinski's Rule of Five through drug scan tool of MOE. This rule gives the explanation about the different properties of drug like absorption metabolism and secretion of drug in human body and it assess the drug on the base of its molecular weight hydrogen acceptors, hydrogen donors and $\log P$ value. All selected molecules contained the properties a drug should have and fulfilled the criteria of Lipinski's Rule. Drug like properties of best molecules are given in (Table I).

\section{Discussion}

Breast cancer is known as a death sentence and second major cause of death in world. Ratio of breast cancer in is one in nine in case of women (Naeem et al., 2008). Main cause of breast cancer is overexpression of estrogen receptor alpha (Hayashi et al., 2003). Therefore ER-a is used as a target for prevention of breast cancer. Tamoxifen is an antagonist of ER-a and commercially available as a drug to control the breast cancer (Jordan, 1992). It binds with Arg394 and blocks the function of estrogen receptor and inhibits the function of ER-a (Desai et al., 2012). In present study tamoxifen was used as a control drug. 


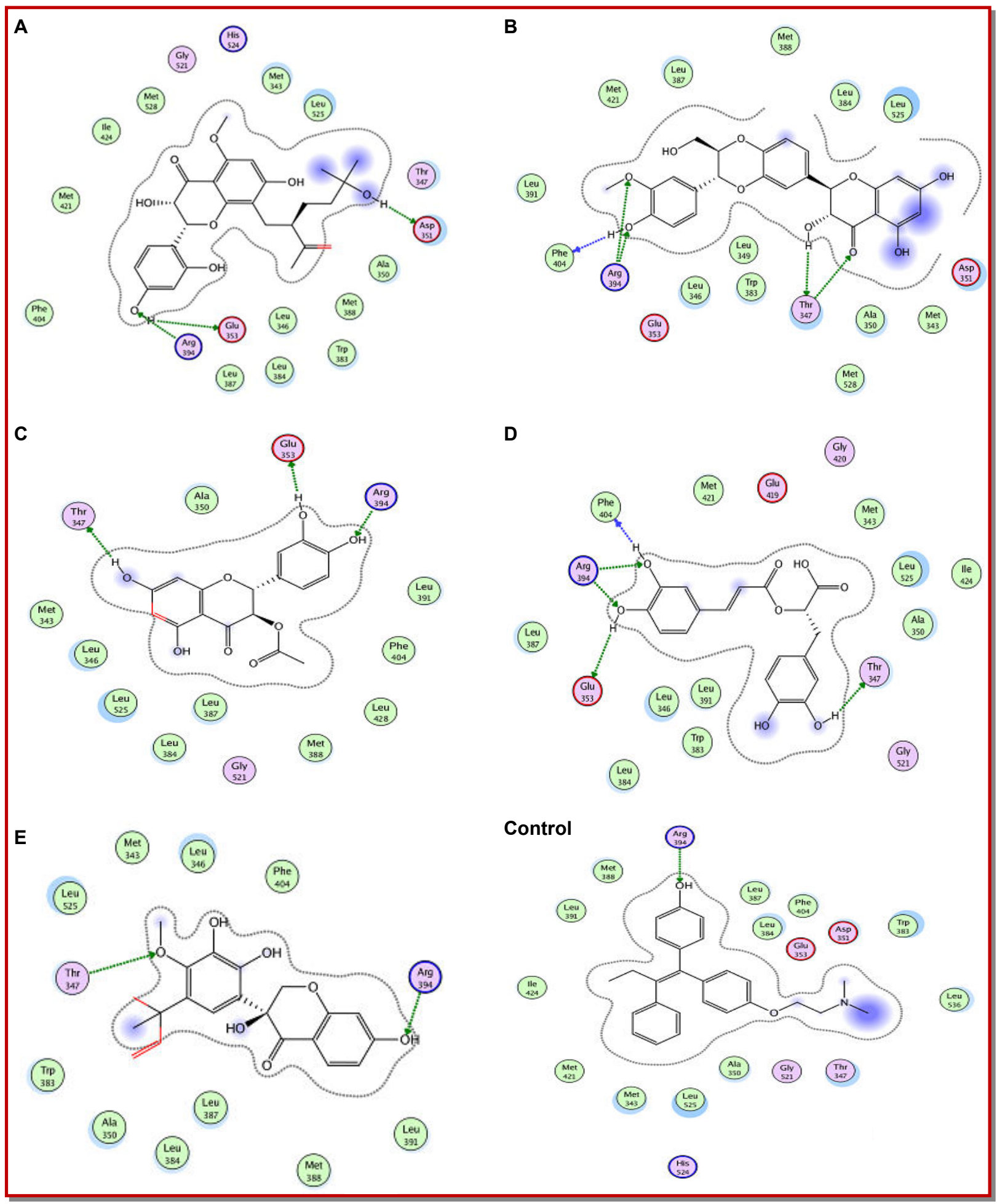

Figure 2: Interactions of top five ligand molecules and control drug (tamoxifen) with estrogen receptor alpha

In recent research, computer aided drug designing (CADD) helps the researcher to decrease the time and money for drug designing projects (Ooms, 2000). Molecular docking is very helpful in studying the interacttions of ligand molecules with the target protein before its in vitro synthesis. Docking is performed through computer programs like MOE (Pedro and Hui, 2008).
4209 ligand molecules taken from different plant sources were docked against the ER-a. All these molecules were taken from ligand database in sdf or mol format and were stored in a database of MOE in $\mathrm{mdb}$ format. All these molecules were docked against the same pocket where reference drug bound. 10 molecules were selected from a library of 4209 molecules 


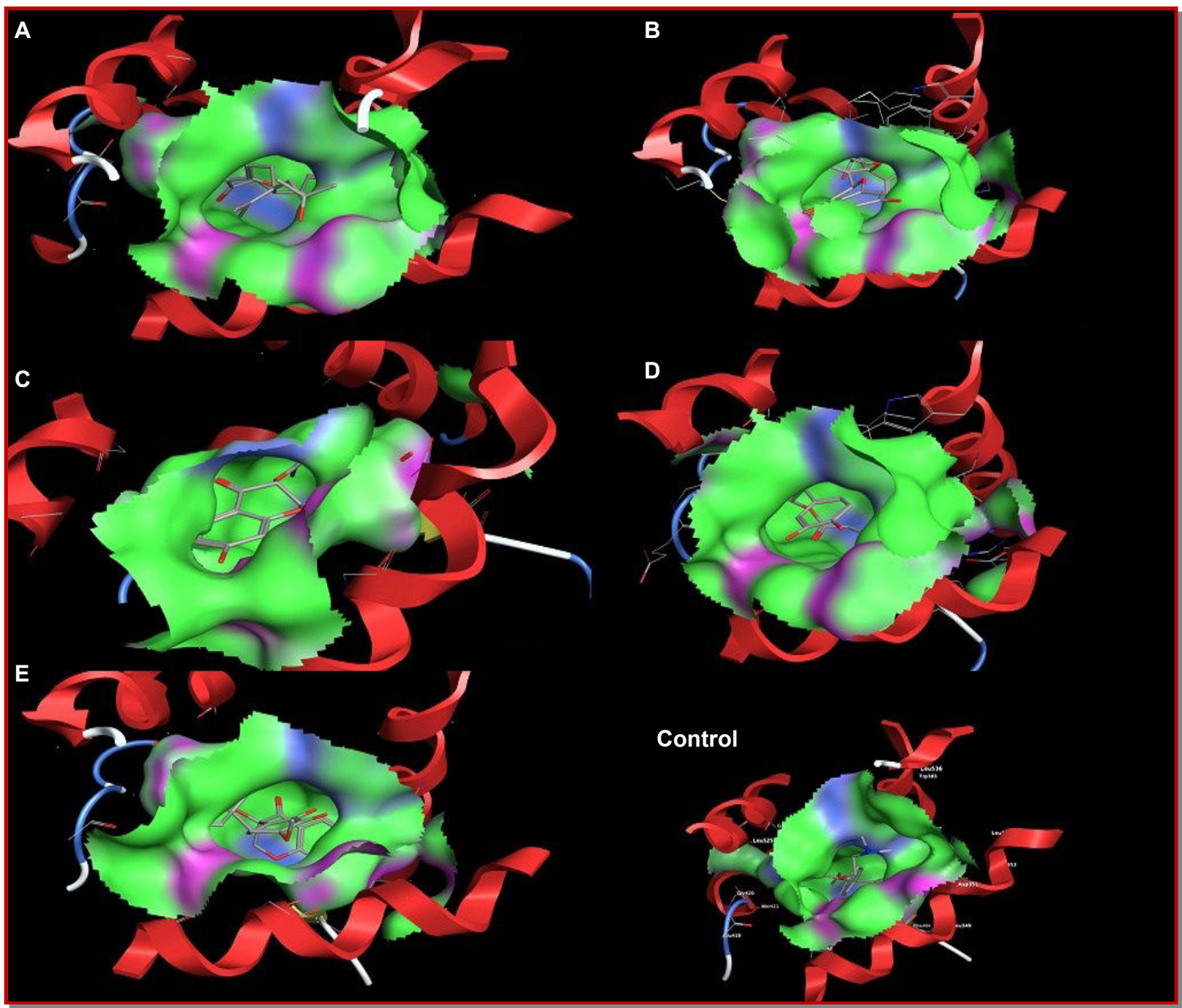

Figure 3: Binding modes of ligand molecules and control within the binding pocket of estrogen receptor alpha

and were further assessed by the interaction analysis. Finalized molecules showed the interactions with the active residue Arg394 and with other residues as well. All the selected molecules were further assessed for Lipinski's Rule of Five. 10 finalized molecules showed the properties which are necessary for a drug candidate.

Present study found the 10 molecules which have less docking store and more stable bonding with the ER-a as compared to the reference drug. It can be said that these selected molecules may be strong antgonists of ER $-\alpha$ as compared to the reference drug. Further study is needed to be conducted to study the other properties of drug like absorption metabolism and excretion in human body.

Ten phytochemicals were selected in this study, which have strong bonding and less docking score as compared to the reference drug (tamoxifen). It can be concluded that these 10 phytochemicals could be used as antagonists of ER- $\alpha$ to prevent the breast cancer in future.

\section{Acknowledgments}

The authors would like to acknowledge Department of Bioinformatics and Biotechnology, Government College University, Faisalabad, 38000, Pakistan.

\section{References}

Ashfaq UA, Mumtaz A, Qamar TU, Fatima T. MAPS Database: Medicinal plant activities, phytochemical and structural database. Bioinformation 2013; 9: 993-95.

Jordan VC. The role of tamoxifen in the treatment and prevention of breast cancer: Current problems in cancer. Curr Prob Cancer. 1992; 16: 134-76.

Desai N, Mahto MK, Alekhya B, Naveen C, Bhaskar M. Comparative docking studies of estrogen receptor inhibitors and their binding interaction analysis. Int J Pharm Sci Rev Res. 2012; 16: 91-95.

Pedro F, Hui L. A Systematic Review on computer-aided drug design: Docking and scoring. J Macao Polytechn Inst. 2010; 47-51. 
Giacinti LP, Claudio P, Lopez M, Giordano A. Epigenetic information and estrogen receptor alpha expression in breast cancer. Oncologist 2006; 11: 1-8.

Hayashi S, Eguchi H, Tanimoto K, Yoshida T, Omoto Y, Inoue A, Yoshida N, Yamaguchi Y. The expression and function of estrogen receptor alpha and beta in human breast cancer and its clinical application. Endocr Relat Cancer. 2003; 10: 193-202.

Holst F, Stahl PR, Ruiz C, Hellwinkel O, Jehan Z, Wendland M, Lebeau A, Terracciano L, Al-Kuraya K, Jänicke F. Estrogen receptor alpha (ESR1) gene amplification is frequent in breast cancer. Nat Genet. 2007; 39: 655-60.

Joy P, Thomas J, Mathew S, Skaria BP. Medicinal plants. Trop
Hort. 1998; 2: 449-632.

MOE: Molecular Operating Environment (MOE). Canada, Chemical Computing Group Inc., 2009.

Naeem M, Khan N, Aman Z, Nasir A, Samad A, Khattak A. Pattern of breast cancer: Experience at lady reading hospital, Peshawar. J Ayub Med Coll Abbottabad. 2008; 20: 22-25.

Ooms F. Molecular modeling and computer aided drug design. Examples of their applications in medicinal chemistry. Curr Med Chem. 2000; 7: 141-58.

Sahu S, Raja S, Kathiresan KP. In silico docking analysis of mangrove-derived compounds against breast cancer protein (BRCA1). Int Multidiscip Res J. 2011; 1. 